\title{
Performance Evaluation of Three-Node Tandem Communication Network Model with Feedback for First Two nodes Having Non Homogeneous Poisson Arrivals
}

\author{
G.Naga Satish \\ Research Scholar \\ Adikavi Nannaya \\ University, RJY
}

\author{
Ch.V.Raghavendran \\ Research Scholar \\ Adikavi Nannaya \\ University, RJY
}

\author{
M.V.Rama Sundari \\ Dept of IT \\ GIET \\ RJY
}

\author{
P.Suresh Varma \\ Dept of CS \\ Adikavi Nannaya \\ University \\ RJY
}

\begin{abstract}
Tandem Queues are widely used in mathematical modeling of random processes describing the operation of Manufacturing systems , supply chains, Computer and telecommunication networks. In many of the communication systems the arrivals are time dependent and can be characterized by a non homogeneous Poisson process. In this paper we developed and analyzed three nodes connected in tandem Queue with feedback for the first and second nodes assuming that arrivals follow non homogeneous Poisson process. Using the difference-differential equations and a probability generating function of the number of packets in the buffer connected to the transmitter the System is analyzed. The System performance is analysed by deriving expressions for the performance measures of the network like mean content of the buffers, mean delays through put, transmitter utilization with mathematical illustrations. The sensitivity analysis of the model reveals that the non homogeneous Poisson arrivals and dynamic bandwidth allocation strategy can reduce burstness in buffer and improve quality of service.
\end{abstract}

Keywords: Feedback, Tandem Network, Non homogeneous Poisson process, Performance measures.

\section{INTRODUCTION}

Tandem queuing systems are the simple queuing networks consisting of finite number of nodes in series. Such systems can be used for modeling real-life networks having a linear topology as well as for validation of general decomposition algorithms in networks. Tandem queuing systems have found much interest in the literature. An extensive survey of early papers on tandem queues can be seen in [1]. Most of these papers are devoted to exponential queuing models in steady state. Over the last two decades, interesting analytical results have been obtained in investigations of complicated two-node tandem queues However, such queues are of limited use for modeling of real-life networks [2]. When the buffer size is finite in tandem, there is a possibility that the system could become blocked. The study of tandem queues without buffers with blocking was studied in 1965 by Avi-Itzhak and Yadin [3]. In an open queuing network, packets enter and depart from the network. Whereas, in a closed queuing network, packets neither enter nor depart from the network. Open queuing networks can be further divided into two categories: open feed forward queuing networks and open feedback queuing networks. In an open feed forward queuing network, a packet cannot appear in the same queue for more than one time. In an open feedback queuing network, after a packet is served by a node, it may reenter the buffer of the same node. Feedback queues play an important role in real-life service systems, where tasks may require repeated services. Tandem queues with feedback have been widely studied in the literature. Communication networks are an important application of such systems. Tang and Zhao, assumed Markovian arrival and service times for feedback queues [4]. On completion of a service at the station, the task either leaves the system with probability $\mathrm{p}$, or goes back, along with all tasks currently waiting in the queue, to the station with probability $1-\mathrm{p}$.

Tandem queues with feedback have comprehensively studied in the literature [5]. Tandem queues with feedback are more complex objects for research than tandem queues without feedback. To improve the quality of service in transmission, several authors have studied the communication networks utilizing tandem queuing analogy [6]. A communication network is analyzed by considering the problems of flow control, routing, bit-dropping, delays, allocation and distribution of data/voice packetization etc., for efficient utilization of the resources. Varying the transmission rates based on the size of the buffer is to be considered for efficient transmission with high quality of service. Some work has been reported in the literature regarding communication networks with dynamic bandwidth allocation/load dependent transmission for improving quality of service by utilizing ideal bandwidth. They considered that the arrivals of messages for transmission are homogeneous [7]. But in many practical situations arising places like satellite communication, wireless communication, telecommunication, computer communication, internet, WAN, the arrival of messages are to be considered as time dependent, in order to have accurate prediction of the performance measures of the system.

One of the most important aspect in developing communication networks is regarding the utilization of congestion control strategies. Usually bit dropping is employed for congestion control. The idea of bit dropping is to discard certain portion of the traffic such as least significant bit in order to reduce the transmission time while maintaining satisfactory quality of service [8]. To improve the quality of service in transmission, several authors have studied the communication networks utilizing tandem queuing analogy [9].Rama Sundari., et al have developed and analyzed a three node communication network model with non homogeneous Poisson arrivals [10] [11]. It is further assumed that transmission time required by each packet at each node is 
dependent on the content of the buffer connected to it. Generally, conducting laboratory experiments with varying load conditions of a communication system in particular with DBA is difficult and complicated. Hence, mathematical models of communication networks are developed to evaluate the performance of the newly proposed communication network model under transient conditions.

Very little work has been reported in literature regarding communication network with non homogeneous Poisson arrivals. Hence, in this paper we develop and analyze a three node communication network model with non homogeneous Poisson arrivals having dynamic bandwidth allocation with feedback to the first and Second nodes. Using differencedifferential equations the performance of the model is analyzed by deriving the joint probability generating function of the number of packets in each buffer. The performance measures like average number of packets in the buffer and in the network; the average waiting time of packets in the buffer and in the network, throughput of the transmitter etc., of the developed network model are derived explicitly. A comparative study of this model with homogeneous Poisson arrivals is also presented. Sensitivity analysis of this model is carried with respect to other parameters. This model is useful for evaluating communication networks where arrivals are time dependent.

\section{THREE NODE TANDEM COMMUNICATION NETWORK MODEL WITH DBA AND NON HOMOGENEOUS POISSON ARRIVALS WITH FEEDBACK FOR FIRST TWO NODES}

We consider an open queuing model of tandem communication network with three nodes. Each node consists of a buffer and a transmitter. The three buffers are Q1, Q2, Q3 and transmitters are S1, S2, S3 connected in tandem. The arrival of packets at the first node follows non homogeneous Poisson processes with a mean arrival rate as a function of $t$ and is in the form of $\lambda(t)=\lambda+\alpha \mathrm{t}$. It is also assumed that the packets are transmitted through the transmitters and the mean service rate in the transmitter is linearly reliant on the content of the buffer connected to it. It is assumed that the packet after getting transmitted through first transmitter may join the second buffer which is in series connected to S2 or may be returned back buffer connected to $\mathrm{S} 1$ and the packets getting transmitted through second transmitter may join the third buffer which is in series connected to S3 or may be retuned back to S2 for retransmission with certain probabilities. The buffers of the nodes follow First-In First-Out (FIFO) technique for transmitting the packets through transmitters. After getting transmitted from the first transmitter the packets are forwarded to Q2 for forward transmission with probability (1- $\theta$ ) or returned back to the Q1 with probability $\theta$ and from the second transmitter the packets are forwarded to Q3 with probability $(1-\pi)$ or returned back to the Q2 with probability $\pi$. The service completion in both the transmitters follows Poisson processes with the parameters $\mu 1, \mu 2$ and $\mu 3$ for the first, second and third transmitters. The transmission rate of each packet is adjusted just before transmission depending on the content of the buffer connected to the transmitter. A schematic diagram representing the network model with three nodes and feedback for first two nodes is shown in figure 2.1

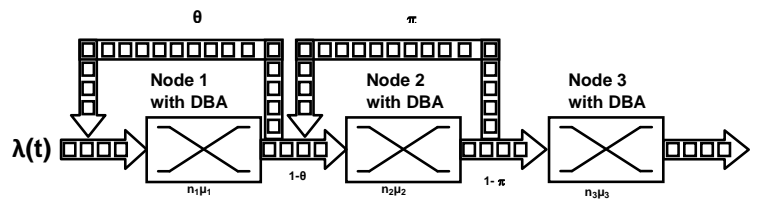

Figure 1: Communication network model with three nodes

Let $n 1$ and $n 2 n 3$ are the number of packets in first, second and third buffers and let $\mathrm{P}_{\mathrm{n} 1 \mathrm{n} 2 \mathrm{n} 3}(\mathrm{t})$ be the probability that there are $\mathrm{n} 1$ packets in the first buffer, $\mathrm{n} 2$ packets in the second buffer and $\mathrm{n} 3$ packets in the third buffer at time t. The difference-differential equations for the above model are as follows:

$$
\begin{aligned}
& \frac{\partial P_{n_{n}, n_{3}}(t)}{\partial t}=-\left(\lambda(t)+n_{1} \mu_{1}(1-\theta)+n_{2} \mu_{2}(1-\pi)+n_{3} \mu_{3}\right) P_{n_{n}, n_{2}, n_{3}}(t)+\lambda(t) P_{n_{1}-1, n_{2}, n_{3}}(t) \\
& +\left(n_{1}+1\right) \mu_{1}(1-\theta) P_{n_{1}+1, n_{2}-1, n_{3}}(t)+\left(n_{2}+1\right) \mu_{2}(1-\pi) P_{n_{1}, n_{2}+1, n_{3}-1}(t)+\left(n_{3}+1\right) \mu_{3} P_{n_{1}, n_{2}, n_{3}+1}(t) \\
& \frac{\partial P_{0, n_{2} n_{3}}(t)}{\partial t}=-\left(\lambda(t)+n_{2} \mu_{2}(1-\pi)+n_{3} \mu_{3}\right) P_{0, n_{2}, n_{3}}(t)+\lambda(t) P_{1, n_{2}, n_{3}}(t)+\mu_{1}(1-\theta) P_{1, n_{2}-1, n_{3}}(t) \\
& +\left(n_{2}+1\right) \mu_{2}(1-\pi) P_{0, n_{2}+1, n_{3}-1}(t)+\left(n_{3}+1\right) \mu_{3} P_{0, n_{2}, n_{3}+1}(t) \\
& \frac{\partial P_{n_{1}, 0, n_{3}}(t)}{\partial t}=-\left(\lambda(t)+n_{1} \mu_{1}(1-\theta)+n_{3} \mu_{3}\right) P_{n_{1}, 0, n_{3}}(t)+\lambda(t) P_{n_{1}-1,0, n_{3}}(t)+ \\
& \mu_{2}(1-\pi) P_{n_{1}, 1, n_{3}-1}(t)+\left(n_{3}+1\right) \mu_{3} P_{n_{1}, 0, n_{3}+1}(t) \\
& \frac{\partial P_{n_{1} n_{2,0}}(t)}{\partial t}=-\left(\lambda(t)+n_{1} \mu_{1}(1-\theta)+n_{2} \mu_{2}(1-\pi)\right) P_{n_{1}, n_{2}, 0}(t)+\lambda(t) P_{n_{1}-1, n_{2}, 0}(t)+ \\
& \left(n_{1}+1\right) \mu_{1}(1-\theta) P_{n_{1}+1, n_{2}-1,0}(t)+\mu_{3} P_{n_{1}, n_{2}, 1}(t) \\
& \frac{\partial P_{0,0, n_{3}}(t)}{\partial t}=-\left(\lambda(t)+n_{3} \mu_{3}\right) P_{0,0, n_{3}}(t)+\mu_{2}(1-\pi) P_{0,1, n_{3}-1}(t)+\left(n_{3}+1\right) \mu_{3} P_{0,0, n_{3}+1}(t) \\
& \frac{\partial P_{0, n_{2}, 0}(t)}{\partial t}=-\left(\lambda(t)+n_{2} \mu_{2}(1-\pi)\right) P_{0, n_{2}, 0}(t)+\mu_{1}(1-\theta) P_{1, n_{2}-1,0}(t)+\mu_{3} P_{0, n_{2}, 1}(t) \\
& \frac{\partial P_{n_{1}, 0,0}(t)}{\partial t}=-\left(\lambda(t)+n_{1} \mu_{1}(1-\theta)\right) P_{n_{1}, 0,0}(t)+\lambda(t) P_{n_{1}-1,0,0}(t)+\mu_{3} P_{n_{1}, 0,1}(t) \\
& \left.\frac{\partial P_{\mathrm{O}, \mathrm{O}, \mathrm{O}}(t)}{\partial t}=-(\lambda(t)) P_{\mathrm{O}, \mathrm{O}, \mathrm{O}}(t)\right)+\mu_{3} P_{\mathrm{O}, \mathrm{O}, 1}(t)
\end{aligned}
$$

Let $\mathrm{P}\left(\mathrm{S}_{1}, \mathrm{~S}_{2}, \mathrm{~S}_{3} ; \mathrm{t}\right)$ be the joint probability generating function of $\mathrm{P}_{\mathrm{n} 1 \mathrm{n} 2 \mathrm{n} 3}(\mathrm{t})$ Then multiply the equation 2.1 with $s_{1}^{n_{1}} S_{2}^{n_{2}} s_{3}^{n_{3}}$ and summing over all $\mathrm{n} 1, \mathrm{n} 2, \mathrm{n} 3$ we get 
$\frac{\partial p(s 1, s 2, s 3: t)}{\partial t}=\sum_{n_{1}=1 n_{2}=\sum_{n_{3}=1}^{\infty}}^{\infty} \sum^{\infty}-\left(\lambda(t)+n_{1} \mu_{1}(1-\theta)+n_{2} \mu_{2}(1-\pi)+n_{3} \mu_{3}\right) P_{n_{1}, n_{2}, n_{3}}(t) s_{1}^{n_{1}} s_{2}^{n_{2}} s_{3}^{n_{3}}$

$+\sum_{n_{1}=1 n_{2}=1 n_{n_{3}}=1}^{\infty} \sum^{\infty} \lambda(t) P_{n_{1}-1, n_{2}, n_{3}}(t) s_{1}^{n_{1}} s_{2}^{n_{2}} s_{3}^{n_{3}}$

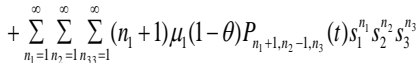

$+\sum_{n_{1}=1 n_{2}=1 n_{3}=1}^{\infty} \sum_{2}^{\infty}\left(n_{2}+1\right) \mu_{2}(1-\pi) P_{n_{1}, n_{2}+1, n_{3}-1}(t) s_{1}^{n_{1}} s_{2}^{n_{2}} s_{3}^{n_{3}}+\sum_{n_{1}=1 n_{2}=1 n_{3}=1}^{\infty} \sum_{3}^{\infty}\left(n_{3}+1\right) \mu_{3} P_{n_{1}, n_{2}, n_{3}+1}(t) s_{1}^{n_{1}} s_{2}^{n_{2}} s_{3}^{n_{3}}$

$+\sum_{n_{2}=1 n_{3}=1}^{\infty}-\left(\lambda(t)+n_{2} \mu_{2}(1-\pi)+n_{3} \mu_{3}\right) P_{0, n_{2}, n_{3}}(t) s_{2}^{n_{2}} s_{3}^{n_{3}}+\sum_{n_{2}=1 n_{3}=1}^{\infty} \sum_{n}^{\infty} \lambda(t) P_{1, n_{2}, n_{3}}(t) s_{2} s_{3}^{n_{2}} s_{3}^{n_{3}}$

$+\sum_{n_{2}=1 n_{3}=1}^{\infty} \sum_{1}^{\infty} \mu_{1}(1-\theta) P_{1, n_{2}-1, n_{3}}(t) s_{2}^{n_{2}} s_{3}^{n_{3}}$

$+\sum_{n_{2}=1 n_{3}=1}^{\infty}\left(n_{2}+1\right) \mu_{2}(1-\pi) P_{n_{1}, n_{2}+1, n_{3}-1}(t) s_{2}^{n_{2}} s_{3}^{n_{3}}+\sum_{n_{2}=1 n_{3}=1}^{\infty}\left(n_{3}+1\right) \mu_{3} P_{0, n_{2}, n_{3}+1}(t) s_{2}^{n_{2}} s_{3}^{n_{3}}$

$+\sum_{n_{1}=1 n_{3}=1}^{\infty} \sum^{\infty}-\left(\lambda(t)+n_{1} \mu_{1}(1-\theta)+n_{3} \mu_{3}\right) P_{n_{1}, 0, n_{3}}(t) s_{1}^{n_{1}} s_{3}^{n_{3}}+\sum_{n_{1}=1 n_{2}=1}^{\infty} \sum_{1}^{\infty} \lambda(t) P_{n_{1}-1,0, n_{3}}(t) s_{1}^{n_{1}} s_{3}^{n_{3}}$

$+\sum_{n_{1}=1}^{\infty} \sum_{n_{3}=1}^{\infty} \mu_{2}(1-\pi) P_{n_{1}, 1, n_{3}-1}(t) s_{1}^{n_{1}} s_{3}^{n_{3}}$

$+\sum_{n_{1}=1 n_{3}=1}^{\infty} \sum_{3}^{\infty}\left(n_{3}+1\right) \mu_{3} P_{n_{1}, 0, n_{3}+1}(t) s_{1}^{n_{1}} s_{3}^{n_{3}}$

$\left.\sum_{n_{1}=1 n_{2}=1}^{\infty} \sum^{\infty}-\left(\lambda(t)+n_{1} \mu_{1}(1-\theta)\right) s_{1}^{n_{1}} s_{2}^{n_{2}}+\sum_{n_{1}=1 n_{2}=1}^{\infty} \sum_{2}^{\infty} n_{2} \mu_{2}(1-\pi)\right) P_{n_{1}, n_{2}, 0}(t) s_{1}^{n_{1}} s_{2}^{n_{2}}$

$+\sum_{n_{1}=1 n_{2}=1}^{\infty} \sum_{1}^{\infty} \lambda(t) P_{n_{1}-1, n_{2}, 0}(t) s_{1}^{n_{1}} s_{2}^{n_{2}}$

$+\sum_{n_{1}=1 n_{2}=1}^{\infty} \sum_{1}^{\infty}\left(n_{1}+1\right) \mu_{1}(1-\theta) P_{n_{1}+1, n_{2}-1,0}(t) s_{1}^{n_{1}} s_{2}^{n_{2}}+\sum_{n_{1}=1 n_{2}=1}^{\infty} \sum_{3}^{\infty} \mu_{n_{1}, n_{2}, 1}(t) s_{1}^{n_{1}} s_{2}^{n_{2}}$

$+\sum_{n_{3}=1}^{\infty}-\left(\lambda(t)+n_{3} \mu_{3}\right) P_{0,0, n_{3}}(t) s_{3}^{n_{3}}+\sum_{n_{3}=1}^{\infty} \mu_{2}(1-\pi) P_{0,1, n_{3}-1}(t) s_{3}^{n_{3}}+\sum_{n_{3}=1}^{\infty}\left(n_{3}+1\right) \mu_{3} P_{0,0, n_{3}+1}(t) s_{3}^{n_{3}}$

$+\sum_{n_{2}=1}^{\infty}-\left(\lambda(t)+n_{2} \mu_{2}(1-\pi)\right) P_{0, n_{2}, 0}(t) s_{2}^{n_{2}}+\sum_{n_{2}=1}^{\infty} \mu_{1}(1-\theta) P_{1, n_{2}-1,0}(t) s_{2}^{n_{2}}+\sum_{n_{2}=1}^{\infty} \mu_{3} P_{0, n_{2}, 1}(t) s_{2}^{n_{2}}$

$+\sum_{n_{1}=1}^{\infty}-\left(\lambda(t)+n_{1} \mu_{1}(1-\theta)\right) P_{n_{1}, 0,0}(t) s_{1}^{n_{1}}+\sum_{n_{1}=1}^{\infty} \lambda(t) P_{n_{1}-1,0,0}(t) s_{1}^{n_{1}}+\sum_{n_{1}=1}^{\infty} \mu_{3} P_{n_{1}, 0,1}(t) s_{1}^{n_{1}}$

$\left.+-(\lambda(t)) P_{0,0,0}(t)\right)+\mu_{3} P_{0,0,1}(t)$

After simplifying we get

$\frac{\partial P\left(s_{1}, s_{2}, s_{3}: t\right)}{\partial t}=\lambda(t) P\left(s_{1}-1\right)+\mu_{1}(1-\theta) \frac{\partial p}{\partial s_{1}}\left(s_{2}-s_{1}\right)+$ $\mu_{2}(1-\pi) \frac{\partial p}{\partial s_{2}}\left(s_{3}-s_{2}\right)+\mu_{3} \frac{\partial p}{\partial s_{3}}\left(1-s_{3}\right)$

Solving equation 2.3 by Lagrangian's method, we get the auxiliary equations as,

$\frac{d t}{1}=\frac{d s_{1}}{\mu_{1}(1-\theta)\left(s_{1}-s_{2}\right)}=\frac{d s_{2}}{\mu_{2}(1-\pi)\left(s_{2}-s_{3}\right)}=\frac{d s_{3}}{\mu_{3}\left(s_{3}-1\right)}=\frac{d p}{\lambda(t) P\left(s_{1}-1\right)}$

To solve the equations in (2.4) the functional form of $\lambda(t)$ is required. Let the mean arrival rate of packets is $\lambda(t)=\lambda+\alpha \mathrm{t}$, where $\lambda>0, \alpha>0$ are constants.

Solving first and fourth terms in equation 2.4 , we get

$a=\left(s_{3}-1\right) e^{-\mu_{3} t}$

Solving first and third terms in equation 2.4, we get

$$
b=\left(s_{2}-1\right) e^{-\mu_{2}(1-\pi) t}+\frac{\left(s_{3}-1\right) \mu_{2}(1-\pi) e^{-\mu_{2}(1-\pi) t}}{\left(\mu_{3}-\mu_{2}(1-\pi)\right)}
$$

Solving first and second terms in equation 2.4 , we get

$$
\begin{aligned}
c= & \left(s_{1}-1\right) e^{-\mu_{1}(1-\theta) t}+\frac{\left(s_{2}-1\right) \mu_{1}(1-\theta) e^{-\mu_{1}(1-\theta) t}}{\left(\mu_{2}(1-\pi)-\mu_{1}(1-\theta)\right)} \\
& +\frac{\left(s_{3}-1\right) \mu_{1}(1-\theta) \mu_{2}(1-\pi) e^{-\mu_{1}(1-\theta) t}}{\left(\mu_{3}-\mu_{1}(1-\theta)\right)\left(\mu_{2}(1-\pi)-\mu_{1}(1-\theta)\right)}
\end{aligned}
$$

Solving first and fifth terms in equation 2.4, we get

$$
\begin{gathered}
d=p \exp \left\{-\left[\frac{\left(s_{1}-1\right)}{\mu_{1}(1-\theta)}\left[\lambda+\alpha t-\frac{\alpha}{\mu_{1}(1-\theta)}\right]+\frac{\left(s_{2}-1\right)}{\mu_{2}(1-\pi)}\left[\lambda+\alpha t-\frac{\alpha\left(\mu_{1}(1-\theta)+\mu_{2}(1-\pi)\right)}{\mu_{1}(1-\theta) \mu_{2}(1-\pi)}\right]+\right.\right. \\
\left.\left.\frac{\left(s_{3}-1\right)}{\mu_{3}}\left[\lambda+\alpha t-\left(\frac{\alpha\left(\mu_{1}(1-\theta) \mu_{2}(1-\pi)+\mu_{2}(1-\pi) \mu_{3}+\mu_{1}(1-\theta) \mu_{3}\right)}{\mu_{1}(1-\theta) \mu_{2}(1-\pi) \mu_{3}}\right)\right]\right\}\right\}
\end{gathered}
$$

Where a,b,c and d are arbitrary constants.

The general solution of equation 2.4 gives the probability generating function of the number of packets in the first and second buffers at time $t$, as $\mathrm{P}\left(\mathrm{S}_{1}, \mathrm{~S}_{2}, \mathrm{~S}_{3} ; \mathrm{t}\right)$.

$$
\begin{aligned}
p\left(s_{1}, s_{2}, s_{3}:\right. & t)=\exp \left\{\frac{s_{1}-1}{\mu_{1}(1-\theta)}\left(\lambda-\frac{\alpha}{\mu_{1}(1-\theta)}\right)\left(1-e^{\mu_{1}(1-\theta) t}\right)\right. \\
& +\frac{s_{1}-1}{\mu_{1}(1-\theta)} \alpha t+\frac{s_{2}-1}{\mu_{2}(1-\pi)}\left(1-e^{\mu_{2}(1-\pi) t}\right)\left(\lambda-\alpha\left(\frac{1}{\mu_{2}(1-\pi)}+\frac{1}{\mu_{1}(1-\theta)}\right)\right) \\
& +\frac{s_{2}-1}{\mu_{2}(1-\pi)} \alpha t+ \\
& \frac{s_{2}-1}{\left(\mu_{2}(1-\pi)-\mu_{1}(1-\theta)\right)}\left(e^{\mu_{2}(1-\pi) t}-e^{\mu_{1}(1-\theta) t}\right)\left(\lambda-\frac{\alpha}{\mu_{1}(1-\theta)}\right) \\
& +\frac{s_{3}-1}{\mu_{3}}\left(1-e^{\mu_{3} t}\right)\left(\lambda-\alpha\left(\frac{1}{\mu_{3}}+\frac{1}{\mu_{1}(1-\theta)}+\frac{1}{\mu_{2}(1-\pi)}\right)\right)+ \\
& \frac{s_{3}-1}{\mu_{3}} \alpha t \\
& +\frac{s_{3}-1}{\left(\mu_{3}-\mu_{2}(1-\pi)\right)}\left(e^{\mu_{3} t}-e^{\mu_{2}(1-\pi) t}\right)\left(\lambda-\alpha\left(\frac{1}{\mu_{2}(1-\pi)}+\frac{1}{\mu_{1}(1-\theta)}\right)\right)+ \\
& \left(s_{3}-1\right) \mu_{2}(1-\pi)\left(\frac{e^{\mu_{1}(1-\theta) t}}{\left(\mu_{1}(1-\theta)-\mu_{3}\right)\left(\mu_{2}(1-\pi)-\mu_{1}(1-\theta)\right)}\right. \\
& +\frac{e^{\mu_{2}(1-\pi) t}}{\left(\mu_{2}(1-\pi)-\mu_{1}(1-\theta)\right)\left(\mu_{3}-\mu_{2}(1-\pi)\right)} \\
& \left.\left.+\frac{e^{\mu_{3} t}}{\left(\mu_{2}(1-\pi)-\mu_{3}\right)\left(\mu_{3}-\mu_{1}(1-\theta)\right)}\right)\left(\lambda-\frac{\alpha}{\mu_{1}(1-\theta)}\right)\right\}
\end{aligned}
$$

\section{PERFORMANCE MEASURES OF THE NETWORK MODEL}

In this section, we derive and analyze the performance measures of the network under transient conditions. Expand $\mathrm{P}\left(\mathrm{S}_{1}, \mathrm{~S}_{2}, \mathrm{~S}_{3} ; \mathrm{t}\right)$ of equation of 2.6 and collect the constant terms. From this, we get the probability that the network is empty as 
$p_{000}(t)=\exp \left\{\frac{-1}{\mu_{1}(1-\theta)}\left(\lambda-\frac{\alpha}{\mu_{1}(1-\theta)}\right)\left(1-e^{\mu_{1}(1-\theta) t}\right)+\frac{-1}{\mu_{1}(1-\theta)} \alpha t\right.$

$+\frac{-1}{\mu_{2}(1-\pi)}\left(1-e^{\mu_{2}(1-\pi) t}\right)\left(\lambda-\alpha\left(\frac{1}{\mu_{2}(1-\pi)}+\frac{1}{\mu_{1}(1-\theta)}\right)\right)+$

$\frac{-1}{\mu_{2}(1-\pi)} \alpha t+\frac{-1}{\left(\mu_{2}(1-\pi)-\mu_{1}(1-\theta)\right)}\left(e^{\mu_{2}(1-\pi) t}-e^{\mu_{1}(1-\theta) t}\right)\left(\lambda-\frac{\alpha}{\mu_{1}(1-\theta)}\right)$

$+\frac{-1}{\mu_{3}}\left(1-e^{\mu_{3}}\right)\left(\lambda-\alpha\left(\frac{1}{\mu_{3}}+\frac{1}{\mu_{1}(1-\theta)}+\frac{1}{\mu_{2}(1-\pi)}\right)\right)+$

$\frac{-1}{\mu_{3}} \alpha t+\frac{-1}{\left(\mu_{3}-\mu_{2}(1-\pi)\right)}\left(e^{\mu_{3} t}-e^{\mu_{2}(1-\pi) t}\right)\left(\lambda-\alpha\left(\frac{1}{\mu_{2}(1-\pi)}+\frac{1}{\mu_{1}(1-\theta)}\right)\right)+$

$(-1) \mu_{2}(1-\pi)\left(\frac{e^{\mu_{1}(1-\theta) t}}{\left(\mu_{1}(1-\theta)-\mu_{3}\right)\left(\mu_{2}(1-\pi)-\mu_{1}(1-\theta)\right)}+\frac{e^{\mu_{2}(1-\pi) t}}{\left(\mu_{2}(1-\pi)-\mu_{1}(1-\theta)\right)\left(\mu_{3}-\mu_{2}(1-\pi)\right)}+\right.$

$\left.\left.\frac{e^{\mu_{3} t}}{\left(\mu_{2}(1-\pi)-\mu_{3}\right)\left(\mu_{3}-\mu_{1}(1-\theta)\right)}\right)\left(\lambda-\frac{\alpha}{\mu_{1}(1-\theta)}\right)\right\}$

Taking $S_{2}, S 3=1$ in equation 2.6 we get probability generating functions of the number of packets in the first buffer is

$P\left(\mathrm{~S}_{1} ; \mathrm{t}\right)=\exp \left\{\frac{S_{1}-1}{\mu_{1}(1-\theta)}\left(\lambda-\frac{\alpha}{\mu_{1}(1-\theta)}\right)\left(1-e^{-\mu_{1}(1-\theta) t}\right)+\frac{S_{1}-1}{\mu_{1}(1-\theta)} \alpha t\right\}$

Probability that the first buffer is empty as $\left(S_{1}=0\right)$

$P_{0 .}(t)=\exp \left\{\frac{-1}{\mu_{1}(1-\theta)}\left(\lambda-\frac{\alpha}{\mu_{1}(1-\theta)}\right)\left(1-e^{\mu_{1}(1-\theta) t}\right)+\frac{1}{\mu_{1}(1-\theta)} \alpha t\right\}$

Taking $S_{1}, S_{3}=1$ in equation 2.6 we get probability generating function of the number of packets in the second buffer is

$P\left(s_{2}: t\right)=\exp \left\{\frac{s_{2}-1}{\mu_{2}(1-\pi)}\left(1-e^{\mu_{2}(1-\pi) t}\right)\left(\lambda-\alpha\left(\frac{1}{\mu_{2}(1-\pi)}+\frac{1}{\mu_{1}(1-\theta)}\right)\right)+\frac{s_{2}-1}{\mu_{2}(1-\pi)} \alpha t+\right.$

$\left.\frac{s_{2}-1}{\left(\mu_{2}(1-\pi)-\mu_{1}(1-\theta)\right)}\left(e^{\mu_{2}(1-\pi) t}-e^{\mu_{1}(1-\theta) t}\right)\left(\lambda-\frac{\alpha}{\mu_{1}(1-\theta)}\right)\right\}$

Probability that the second buffer is empty as $\left(\mathrm{S}_{2}=0\right)$

$P_{\text {o. }}=\exp \left\{\frac{-1}{\mu_{2}(1-\pi)}\left(1-e^{\mu_{2}(1-\pi) t}\right)\left(\lambda-\alpha\left(\frac{1}{\mu_{2}(1-\pi)}+\frac{1}{\mu_{1}(1-\theta)}\right)\right)+\frac{-1}{\mu_{2}(1-\pi)} \alpha t+\right.$

$\left.\frac{-1}{\left(\mu_{2}(1-\pi)-\mu_{1}(1-\theta)\right)}\left(e^{\mu_{2}(1-\pi) t}-e^{\mu_{1}(1-\theta) t}\right)\left(\lambda-\frac{\alpha}{\mu_{1}(1-\theta)}\right)\right\}$

Taking $s_{1}=1$ and $s_{2}=1$ we get we get probability generating function of the no of packets in the third buffer

$$
\begin{aligned}
& P\left(s_{3}: t\right)=\exp \left\{\frac{s_{3}-1}{\mu_{3}}\left(1-e^{\mu_{3} t}\right)\left(\lambda-\alpha\left(\frac{1}{\mu_{3}}+\frac{1}{\mu_{1}(1-\theta)}+\frac{1}{\mu_{2}(1-\pi)}\right)\right)+\frac{s_{3}-1}{\mu_{3}} \alpha t+\right. \\
& \frac{s_{3}-1}{\left(\mu_{3}-\mu_{2}(1-\pi)\right)}\left(e^{\mu_{3} t}-e^{\mu_{2}(1-\pi) t}\right)\left(\lambda-\alpha\left(\frac{1}{\mu_{2}(1-\pi)}+\frac{1}{\mu_{1}(1-\theta)}\right)+\right. \\
& \left(s_{3}-1\right) \mu_{2}(1-\pi)\left(\frac{e^{\mu_{1}(1-\theta) t}}{\left(\mu_{1}(1-\theta)-\mu_{3}\right)\left(\mu_{2}(1-\pi)-\mu_{1}(1-\theta)\right)}+\frac{e^{\mu_{2}(1-\pi) t}}{\left(\mu_{2}(1-\pi)-\mu_{1}(1-\theta)\right)\left(\mu_{3}-\mu_{2}(1-\pi)\right)}+\right. \\
& \left.\left.\frac{e^{\mu_{3} t}}{\left(\mu_{2}(1-\pi)-\mu_{3}\right)\left(\mu_{3}-\mu_{1}(1-\theta)\right)}\right)\left(\lambda-\frac{\alpha}{\mu_{1}(1-\theta)}\right)\right\}
\end{aligned}
$$

Probability that the third buffer is empty $\left(S_{3}=0\right)$

$$
\begin{aligned}
P_{.0}= & \exp \left\{\frac{-1}{\mu_{3}}\left(1-e^{\mu_{3} t}\right)\left(\lambda-\alpha\left(\frac{1}{\mu_{3}}+\frac{1}{\mu_{1}(1-\theta)}+\frac{1}{\mu_{2}(1-\pi)}\right)\right)+\frac{-1}{\mu_{3}} \alpha t+\right. \\
& \frac{-1}{\left(\mu_{3}-\mu_{2}(1-\pi)\right)}\left(e^{\mu_{3} t}-e^{\mu_{2}(1-\pi) t}\right)\left(\lambda-\alpha\left(\frac{1}{\mu_{2}(1-\pi)}+\frac{1}{\mu_{1}(1-\theta)}\right)\right)+ \\
& \frac{(-1) \mu_{2}(1-\pi)\left(\frac{e^{\mu_{1}(1-\theta) t}}{\left(\mu_{1}(1-\theta)-\mu_{3}\right)\left(\mu_{2}(1-\pi)-\mu_{1}(1-\theta)\right)}+\right.}{\left(\mu_{2}(1-\pi)-\mu_{1}(1-\theta)\right)\left(\mu_{3}-\mu_{2}(1-\pi)\right)}+ \\
& \left.\left.\frac{e^{\mu_{2}(1-\pi) t}}{\left(\mu_{2}(1-\pi)-\mu_{3}\right)\left(\mu_{3}-\mu_{1}(1-\theta)\right)}\right)\left(\lambda-\frac{\alpha}{\mu_{1}(1-\theta)}\right)\right\}
\end{aligned}
$$

Mean Number of Packets in the First Buffer is

$$
\begin{aligned}
L_{1}(t) & =\frac{\partial p(s 1: t)}{\partial s_{1}}=\frac{1}{\mu_{1}(1-\theta)}\left(\lambda-\frac{\alpha}{\mu_{1}(1-\theta)}\right)\left(1-e^{\mu_{1}(1-\theta) t}\right) \\
& +\frac{1}{\mu_{1}(1-\theta)} \alpha t
\end{aligned}
$$

Utilization of the first transmitter is

$$
U_{1}(t)=1-P_{0 . .}(t)=1-\exp \left\{\frac{-1}{\mu_{1}(1-\theta)}\left(\lambda-\frac{\alpha}{\mu_{1}(1-\theta)}\right)\left(1-e^{\mu_{1}(1-\theta) t}\right)+\frac{-1}{\mu_{1}(1-\theta)} \alpha t\right\}
$$

Variance of the Number of packets in the first buffer is

$$
V_{1}(t)=\frac{1}{\mu_{1}(1-\theta)}\left(\lambda-\frac{\alpha}{\mu_{1}(1-\theta)}\right)\left(1-e^{\mu_{1}(1-\theta) t}\right)+\frac{1}{\mu_{1}(1-\theta)} \alpha t
$$

Throughput of the first transmitter is

$T h_{1}=\mu_{1}\left(1-P_{0 . .}(t)\right)=\mu_{1}\left(1+\exp \left\{\frac{1}{\mu_{1}(1-\theta)}\left(\lambda-\frac{\alpha}{\mu_{1}(1-\theta)}\right)\left(1-e^{\mu_{1}(1-\theta) t}\right)+\frac{1}{\mu_{1}(1-\theta)} \alpha t\right\}\right)$

Average waiting time in the first Buffer is

$$
W_{1}(t)=\frac{L_{1}(t)}{\mu_{1}\left(1-P_{0 . .}(t)\right)}
$$

Mean number of packets in the second buffer is

$$
\begin{aligned}
L_{2}(t)= & \frac{\partial p\left(s_{2}: t\right)}{\partial s_{2}}=\frac{1}{\mu_{2}(1-\pi)}\left(1-e^{\mu_{2}(1-\pi) t}\right)\left(\lambda-\alpha\left(\frac{1}{\mu_{2}(1-\pi)}+\frac{1}{\mu_{1}(1-\theta)}\right)\right)+\frac{1}{\mu_{2}(1-\pi)} \alpha t+ \\
& \frac{1}{\left(\mu_{2}(1-\pi)-\mu_{1}(1-\theta)\right)}\left(e^{\mu_{2}(1-\pi) t}-e^{\mu_{1}(1-\theta) t}\right)\left(\lambda-\frac{\alpha}{\mu_{1}(1-\theta)}\right)
\end{aligned}
$$

Utilization of the second transmitter is

$$
\begin{gathered}
U_{2}(t)=1-P_{.0 .}(t)=1-\exp \left\{\frac{-1}{\mu_{2}(1-\pi)}\left(1-e^{\mu_{2}(1-\pi) t}\right)\left(\lambda-\alpha\left(\frac{1}{\mu_{2}(1-\pi)}+\frac{1}{\mu_{1}(1-\theta)}\right)\right)+\right. \\
\left.\frac{-1}{\mu_{2}(1-\pi)} \alpha t+\frac{-1}{\left(\mu_{2}(1-\pi)-\mu_{1}(1-\theta)\right)}\left(e^{\mu_{2}(1-\pi) t}-e^{\mu_{1}(1-\theta) t}\right)\left(\lambda-\frac{\alpha}{\mu_{1}(1-\theta)}\right)\right\}
\end{gathered}
$$

Variance of the number of packets in the second buffer is

$$
\begin{aligned}
V_{2}(t)= & \left\{\frac { 1 } { \mu _ { 2 } ( 1 - \pi ) } \left(1-e^{\mu_{2}(1-\pi) t}\left(\lambda-\alpha\left(\frac{1}{\mu_{2}(1-\pi)}+\frac{1}{\mu_{1}(1-\theta)}\right)\right)+\right.\right. \\
& \left.\frac{1}{\mu_{2}(1-\pi)} \alpha t+\frac{1}{\left(\mu_{2}(1-\pi)-\mu_{1}(1-\theta)\right)}\left(e^{\mu_{2}(1-\pi) t}-e^{\mu_{1}(1-\theta) t}\right)\left(\lambda-\frac{\alpha}{\mu_{1}(1-\theta)}\right)\right\}
\end{aligned}
$$


Throughput of the second transmitter is

$T h_{2}(t)=\mu_{2}\left(1-P_{0}(t)\right)$

$$
\begin{aligned}
& =\mu_{2}\left(1+\exp \left\{\frac{1}{\mu_{2}(1-\pi)}\left(1-e^{\mu_{2}(1-\pi) t}\right)\left(\lambda-\alpha\left(\frac{1}{\mu_{2}(1-\pi)}+\frac{1}{\mu_{1}(1-\theta)}\right)\right)\right.\right. \\
& \left.\left.+\frac{1}{\mu_{2}(1-\pi)} \alpha t+\frac{1}{\left(\mu_{2}(1-\pi)-\mu_{1}(1-\theta)\right)}\left(e^{\mu_{2}(1-\pi) t}-e^{\mu_{1}(1-\theta) t}\right)\left(\lambda-\frac{\alpha}{\mu_{1}(1-\theta)}\right)\right\}\right)
\end{aligned}
$$

Average waiting time in the second buffer is

$$
W_{2}(t)=\frac{L_{2}(t)}{\mu_{2}\left(1-P_{.0 .}\right)}
$$

The mean number of packets in the Third buffer is

$$
\begin{aligned}
& L_{3}(t)=\frac{\partial p\left(s_{3}: t\right)}{\partial s_{3}}=\left\{\frac{1}{\mu_{3}}\left(1-e^{\mu_{3} t}\right)\left(\lambda-\alpha\left(\frac{1}{\mu_{3}}+\frac{1}{\mu_{1}(1-\theta)}+\frac{1}{\mu_{2}(1-\pi)}\right)\right)+\right. \\
& \frac{1}{\mu_{3}} \alpha t+\frac{1}{\left(\mu_{3}-\mu_{2}(1-\pi)\right)}\left(e^{\mu_{3} t}-e^{\mu_{2}(1-\pi) t}\right)\left(\lambda-\alpha\left(\frac{1}{\mu_{2}(1-\pi)}+\frac{1}{\mu_{1}(1-\theta)}\right)\right)+ \\
& \mu_{2}(1-\pi)\left(\frac{e^{\mu_{1}(1-\theta) t}}{\frac{\left(\mu_{1}(1-\theta)-\mu_{3}\right)\left(\mu_{2}(1-\pi)-\mu_{1}(1-\theta)\right)}{\left(\mu_{2}(1-\pi) t\right.}}+\frac{e^{\mu_{3} t}}{\left(\mu_{2}(1-\pi)-\mu_{1}(1-\theta)\right)\left(\mu_{3}-\mu_{2}(1-\pi)\right)}+\left(\lambda-\frac{\alpha}{\mu_{1}(1-\theta)}\right)\right\}
\end{aligned}
$$

Utilization of the Third Transmitter is

$$
\begin{aligned}
& U_{3}(t)=1-P_{.0}=1-\exp \left\{\frac { - 1 } { \mu _ { 3 } } \left(1-e^{\mu_{3} t}\left(\lambda-\alpha\left(\frac{1}{\mu_{3}}+\frac{1}{\mu_{1}(1-\theta)}+\frac{1}{\mu_{2}(1-\pi)}\right)\right)+\frac{-1}{\mu_{3}} \alpha t+\right.\right. \\
& \frac{-1}{\left(\mu_{3}-\mu_{2}(1-\pi)\right)}\left(e^{\mu_{3} t}-e^{\mu_{2}(1-\pi) t}\left(\lambda-\alpha\left(\frac{1}{\mu_{2}(1-\pi)}+\frac{1}{\mu_{1}(1-\theta)}\right)\right)+\right. \\
& (-1) \mu_{2}(1-\pi)\left(\frac{e^{\mu_{1}(1-\theta) t}}{\left(\frac{\left.\mu_{1}(1-\theta)-\mu_{3}\right)\left(\mu_{2}(1-\pi)-\mu_{1}(1-\theta)\right)}{\mu_{2}}+\frac{e^{\mu_{2}(1-\pi) t}}{\left(\mu_{2}(1-\pi)-\mu_{1}(1-\theta)\right)\left(\mu_{3}-\mu_{2}(1-\pi)\right)}+\left(\lambda-\frac{\alpha}{\mu_{1}(1-\theta)}\right)\right\}}\right.
\end{aligned}
$$

Variance of the number of packets in the Third buffer is

$$
\begin{aligned}
& V_{3}(t)=\left\{\frac{1}{\mu_{3}}\left(1-e^{\mu_{3}}\right\}\left(\lambda-\alpha\left(\frac{1}{\mu_{3}}+\frac{1}{\mu_{1}(1-\theta)}+\frac{1}{\mu_{2}(1-\pi)}\right)\right)+\frac{1}{\mu_{3}} \alpha t+\frac{1}{\left(\mu_{3}-\mu_{2}(1-\pi)\right)}\left(e^{\mu_{2} t}-e^{\mu_{2}(1-\pi) t}\right)\left(\lambda-\alpha\left(\frac{1}{\mu_{2}(1-\pi)}+\frac{1}{\mu_{1}(1-\theta)}\right)+\right.\right. \\
& \left.\mu_{2}(1-\pi)\left(\frac{e^{\mu_{1}(1-\theta) t}}{\left(\mu_{1}(1-\theta)-\mu_{3}\right)\left(\mu_{2}(1-\pi)-\mu_{1}(1-\theta)\right)}+\frac{e^{\mu_{1}(1-\pi) t}}{\left(\mu_{2}(1-\pi)-\mu_{1}(1-\theta)\right)\left(\mu_{3}-\mu_{2}(1-\pi)\right)}+\frac{e^{\mu_{3} t}}{\left(\mu_{2}(1-\pi)-\mu_{3}\right)\left(\mu_{3}-\mu_{1}(1-\theta)\right)}\right)\left(\lambda-\frac{\alpha}{\mu_{1}(1-\theta)}\right)\right\}
\end{aligned}
$$

Through put of the Third Transmitter is

Table 4.1 Values of Utilization and Throughput of the Network model with DBA and Non-Homogeneous Poisson arrivals

\begin{tabular}{|r|c|c|c|c|c|c|c|c|c|c|c|c|c|}
\hline $\mathrm{t}$ & $\lambda$ & $\alpha$ & $\theta$ & $\pi$ & $\mu_{1}$ & $\mu_{2}$ & $\mu_{3}$ & $\mathrm{U}_{1}(\mathrm{t})$ & $\mathrm{U}_{2}(\mathrm{t})$ & $\mathrm{U}_{3}(\mathrm{t})$ & $\mathrm{Th}_{1}(\mathrm{t})$ & $\mathrm{Th}_{2}(\mathrm{t})$ & $\mathrm{Th}_{3}(\mathrm{t})$ \\
\hline $\mathbf{0 . 1}$ & 2 & 1 & 0.1 & 0.1 & 5 & 15 & 25 & 0.1524 & 0.0258 & 0.0076 & 0.7621 & 0.3871 & 0.1906 \\
\hline $\mathbf{0 . 3}$ & 2 & 1 & 0.1 & 0.1 & 5 & 15 & 25 & 0.3018 & 0.0938 & 0.0452 & 1.5092 & 1.4065 & 1.1305 \\
\hline $\mathbf{0 . 5}$ & 2 & 1 & 0.1 & 0.1 & 5 & 15 & 25 & 0.3716 & 0.1328 & 0.0703 & 1.8579 & 1.9922 & 1.7585 \\
\hline $\mathbf{0 . 7}$ & 2 & 1 & 0.1 & 0.1 & 5 & 15 & 25 & 0.4136 & 0.1560 & 0.0851 & 2.0678 & 2.3399 & 2.1284 \\
\hline $\mathbf{0 . 9}$ & 2 & 1 & 0.1 & 0.1 & 5 & 15 & 25 & 0.4447 & 0.1726 & 0.0954 & 2.2233 & 2.5885 & 2.3854 \\
\hline 0.5 & $\mathbf{3}$ & 1 & 0.1 & 0.1 & 5 & 15 & 25 & 0.4849 & 0.1853 & 0.0999 & 2.4243 & 2.7792 & 2.4981 \\
\hline 0.5 & $\mathbf{4}$ & 1 & 0.1 & 0.1 & 5 & 15 & 25 & 0.5777 & 0.2346 & 0.1286 & 2.8887 & 3.5185 & 3.2141 \\
\hline 0.5 & $\mathbf{5}$ & 1 & 0.1 & 0.1 & 5 & 15 & 25 & 0.6539 & 0.2809 & 0.1563 & 3.2693 & 4.2131 & 3.9074 \\
\hline 0.5 & $\mathbf{6}$ & 1 & 0.1 & 0.1 & 5 & 15 & 25 & 0.7163 & 0.3244 & 0.1831 & 3.5814 & 4.8657 & 4.5786 \\
\hline 0.5 & $\mathbf{7}$ & 1 & 0.1 & 0.1 & 5 & 15 & 25 & 0.7674 & 0.3653 & 0.2091 & 3.8371 & 5.4788 & 5.2284 \\
\hline 0.5 & 2 & $\mathbf{0}$ & 0.1 & 0.1 & 5 & 15 & 25 & 0.3281 & 0.1173 & 0.0626 & 1.6403 & 1.7601 & 1.5658 \\
\hline 0.5 & 2 & $\mathbf{0 . 5}$ & 0.1 & 0.1 & 5 & 15 & 25 & 0.3502 & 0.1251 & 0.0665 & 1.7509 & 1.8766 & 1.6623 \\
\hline 0.5 & 2 & $\mathbf{1}$ & 0.1 & 0.1 & 5 & 15 & 25 & 0.3716 & 0.1328 & 0.0703 & 1.8579 & 1.9922 & 1.7585 \\
\hline 0.5 & 2 & $\mathbf{1 . 5}$ & 0.1 & 0.1 & 5 & 15 & 25 & 0.3923 & 0.1404 & 0.0742 & 1.9613 & 2.1067 & 1.8543 \\
\hline 0.5 & 2 & $\mathbf{2}$ & 0.1 & 0.1 & 5 & 15 & 25 & 0.4123 & 0.1480 & 0.0780 & 2.0613 & 2.2203 & 1.9496 \\
\hline 0.5 & 2 & 1 & $\mathbf{0 . 1}$ & 0.1 & 5 & 15 & 25 & 0.3716 & 0.1328 & 0.0703 & 1.8579 & 1.9922 & 1.7585 \\
\hline
\end{tabular}

\section{PERFORMANCE EVALUATION OF THE NETWORK MODEL}

In this section, the performance of the network model is discussed with numerical illustration. Different values of the parameters are taken for bandwidth allocation and arrival of packets. The packet arrival $(\lambda)$ varies from $2 \times 10^{4}$ packets/sec to $7 \times 10^{4}$ packets/sec, probability parameters $(\theta, \pi)$ varies from 0.1 to 0.9 , the transmission rate for first transmitter $\left(\mu_{1}\right)$ varies from $5 \times 10^{4}$ packets/sec to $9 \times 10^{4}$ packets/sec, transmission rate for second transmitter $\left(\mu_{2}\right)$ varies from $15 \times 10^{4}$ packets/sec to $19 \times 10^{4}$ packets/sec and transmission rate for third transmitter $\left(\mu_{3}\right)$ varies from $25 \times 10^{4}$ packets/sec to $29 \times 10^{4}$ packets $/ \mathrm{sec}$. Dynamic Bandwidth Allocation strategy is considered for both the two transmitters. So, the transmission rate of each packet depends on the number of packets in the buffer connected to corresponding transmitter.

The equations 3.9, 3.11, 3.14, 3.16, 3.19 and 3.21 are used for computing the utilization of the transmitters and throughput of the transmitters for different values of parameters $t, \lambda, \alpha, \theta, \pi$, $\mu_{1}, \mu_{2}, \mu_{3}$ and the results are presented in the Table 4.1. The Graphs in figure 4.1 shows the relationship between utilization of the transmitters and throughput of the transmitters.

Average waiting in third buffer is

Mean number of packets in the entire network at time $t$ is 


\begin{tabular}{|l|l|l|l|l|l|l|l|l|l|l|l|l|l|}
0.5 & 2 & 1 & $\mathbf{0 . 3}$ & 0.1 & 5 & 15 & 25 & 0.4216 & 0.1209 & 0.0633 & 2.1081 & 1.8128 & 1.5820 \\
\hline 0.5 & 2 & 1 & $\mathbf{0 . 5}$ & 0.1 & 5 & 15 & 25 & 0.4814 & 0.1028 & 0.0530 & 2.4070 & 1.5414 & 1.3255 \\
\hline 0.5 & 2 & 1 & $\mathbf{0 . 7}$ & 0.1 & 5 & 15 & 25 & 0.5517 & 0.0749 & 0.0379 & 2.7586 & 1.1235 & 0.9477 \\
\hline 0.5 & 2 & 1 & $\mathbf{0 . 9}$ & 0.1 & 5 & 15 & 25 & 0.6321 & 0.0311 & 0.0153 & 3.1606 & 0.4660 & 0.3831 \\
\hline 0.5 & 2 & 1 & 0.1 & $\mathbf{0 . 1}$ & 5 & 15 & 25 & 0.3716 & 0.1328 & 0.0703 & 1.8579 & 1.9922 & 1.7585 \\
\hline 0.5 & 2 & 1 & 0.1 & $\mathbf{0 . 3}$ & 5 & 15 & 25 & 0.3716 & 0.1625 & 0.0678 & 1.8579 & 2.4376 & 1.6954 \\
\hline 0.5 & 2 & 1 & 0.1 & $\mathbf{0 . 5}$ & 5 & 15 & 25 & 0.3716 & 0.2072 & 0.0630 & 1.8579 & 3.1087 & 1.5754 \\
\hline 0.5 & 2 & 1 & 0.1 & $\mathbf{0 . 7}$ & 5 & 15 & 25 & 0.3716 & 0.2861 & 0.0480 & 1.8579 & 4.2916 & 1.1999 \\
\hline 0.5 & 2 & 1 & 0.1 & $\mathbf{0 . 9}$ & 5 & 15 & 25 & 0.3716 & 0.3975 & 0.0268 & 1.8579 & 5.9629 & 0.6712 \\
\hline 0.5 & 2 & 1 & 0.1 & 0.1 & $\mathbf{5}$ & 15 & 25 & 0.3716 & 0.1328 & 0.0703 & 1.8579 & 1.9922 & 1.7585 \\
\hline 0.5 & 2 & 1 & 0.1 & 0.1 & $\mathbf{6}$ & 15 & 25 & 0.3337 & 0.1402 & 0.0748 & 2.0025 & 2.1027 & 1.8710 \\
\hline 0.5 & 2 & 1 & 0.1 & 0.1 & $\mathbf{7}$ & 15 & 25 & 0.3017 & 0.1454 & 0.0781 & 2.1119 & 2.1811 & 1.9532 \\
\hline 0.5 & 2 & 1 & 0.1 & 0.1 & $\mathbf{8}$ & 15 & 25 & 0.2745 & 0.1492 & 0.0806 & 2.1959 & 2.2374 & 2.0138 \\
\hline 0.5 & 2 & 1 & 0.1 & 0.1 & $\mathbf{9}$ & 15 & 25 & 0.2513 & 0.1519 & 0.0824 & 2.2613 & 2.2784 & 2.0591 \\
\hline 0.5 & 2 & 1 & 0.1 & 0.1 & 5 & $\mathbf{1 5}$ & 25 & 0.3716 & 0.1328 & 0.0703 & 1.8579 & 1.9922 & 1.7585 \\
\hline 0.5 & 2 & 1 & 0.1 & 0.1 & 5 & $\mathbf{1 6}$ & 25 & 0.3716 & 0.1258 & 0.0709 & 1.8579 & 2.0132 & 1.7716 \\
\hline 0.5 & 2 & 1 & 0.1 & 0.1 & 5 & $\mathbf{1 7}$ & 25 & 0.3716 & 0.1195 & 0.0713 & 1.8579 & 2.0317 & 1.7829 \\
\hline 0.5 & 2 & 1 & 0.1 & 0.1 & 5 & $\mathbf{1 8}$ & 25 & 0.3716 & 0.1138 & 0.0717 & 1.8579 & 2.0481 & 1.7928 \\
\hline 0.5 & 2 & 1 & 0.1 & 0.1 & 5 & $\mathbf{1 9}$ & 25 & 0.3716 & 0.1086 & 0.0721 & 1.8579 & 2.0627 & 1.8014 \\
\hline 0.5 & 2 & 1 & 0.1 & 0.1 & 5 & 15 & $\mathbf{2 5}$ & 0.3716 & 0.1328 & 0.0703 & 1.8579 & 1.9922 & 1.7585 \\
\hline 0.5 & 2 & 1 & 0.1 & 0.1 & 5 & 15 & $\mathbf{2 6}$ & 0.3716 & 0.1328 & 0.0679 & 1.8579 & 1.9922 & 1.7649 \\
\hline 0.5 & 2 & 1 & 0.1 & 0.1 & 5 & 15 & $\mathbf{2 7}$ & 0.3716 & 0.1328 & 0.0656 & 1.8579 & 1.9922 & 1.7709 \\
\hline 0.5 & 2 & 1 & 0.1 & 0.1 & 5 & 15 & $\mathbf{2 8}$ & 0.3716 & 0.1328 & 0.0634 & 1.8579 & 1.9922 & 1.7764 \\
\hline 0.5 & 2 & 1 & 0.1 & 0.1 & 5 & 15 & $\mathbf{2 9}$ & 0.3716 & 0.1328 & 0.0614 & 1.8579 & 1.9922 & 1.7816 \\
\hline
\end{tabular}

From the table 4.1 it is observed that, when the time $(t)$ and $\lambda$ increases, the utilization of the transmitters increases for the fixed value of other parameters $\theta, \pi, \mu_{1}, \mu_{2}$. As the arrivals of the packets at the first node is non-homogeneous Poisson in nature, when the value of $\alpha$ increase from 0 to 2 , the utilization, throughput of the first, second, third transmitters increases for fixed values of other parameters. As the transmitter probability parameter $\theta$ increases from 0.1 to 0.9 , the utilization of first transmitter increases and utilization of the second, third transmitter decreases, this is due to the number of packets arriving at the third transmitter are decreasing as number of packets going back to the first and second transmitter in feedback are increasing. As the transmitter probability parameter $\pi$ increases from 0.1 to 0.9 , the utilization of first transmitter remains constant, utilization of the second transmitter increases and the utilization of third transmitter decreases. This is because the number of packets arriving at the second transmitter is packets arriving directly from the first transmitter and packets arrived for retransmission in feedback and the decrease at the third transmitter is because of packets going back to the second transmitter in feedback is increasing. As the transmission rate of the first transmitter $\left(\mu_{1}\right)$ increases from 5 to 9 , the utilization of the first transmitter decreases and the utilization of the second transmitter and third transmitter increases by keeping the other parameters as constant. As the transmission rate of the second transmitter $\left(\mu_{2}\right)$ increases from 15 to 19 , the utilization of the first transmitter is constant and the utilization of the second transmitter decreases, the utilization of the third transmitter increases by keeping the other parameters as constant. As the transmission rate of the third transmitter $\left(\mu_{3}\right)$ increases from 25 to 29 the utilization of the first and second transmitters is constant and the utilization of the third transmitter decreases by keeping the other parameters as constant. It is also observed from the table 4.1 that, as the time (t) increases, the throughput of first, second and third transmitters is increasing for the fixed values of other parameters. When the parameter $\lambda$ increases from $3 \times 10^{4}$ packets $/ \mathrm{sec}$ to $7 \times 10^{4}$ packets $/ \mathrm{sec}$, the throughput of three transmitters increases. As the parameter $\alpha$ increases, the throughput of transmitters also increases. As the first probability parameter $\theta$ value increases from 0.1 to 0.9 , the throughput of the first transmitter increases and the throughput of the second and third transmitter decreases. As the second probability parameter $\pi$ value increases from 0.1 to 0.9 , the throughput of the first transmitter remains constant, throughput of the second transmitter increases and throughput of the third transmitter decreases. As the transmission rate of the first transmitter $\left(\mu_{1}\right)$ increases from $5 \times 10^{4}$ packets/sec to $9 \times 10^{4}$ packets/sec, the throughput of the first, second and third transmitters increases. The transmission rate of the second transmitter $\left(\mu_{2}\right)$ increases from $15 \times 10^{4}$ packets/sec to $19 \times 10^{4}$ packets/sec and the throughput of the first transmitter is constant and the throughput of the second, third transmitter increases. The transmission rate of the third transmitter $\left(\mu_{3}\right)$ increases from $25 \times 10^{4}$ to $29 \times 10^{4}$ the throughput of the first, second transmitter is constant and throughput of third transmitter increases.
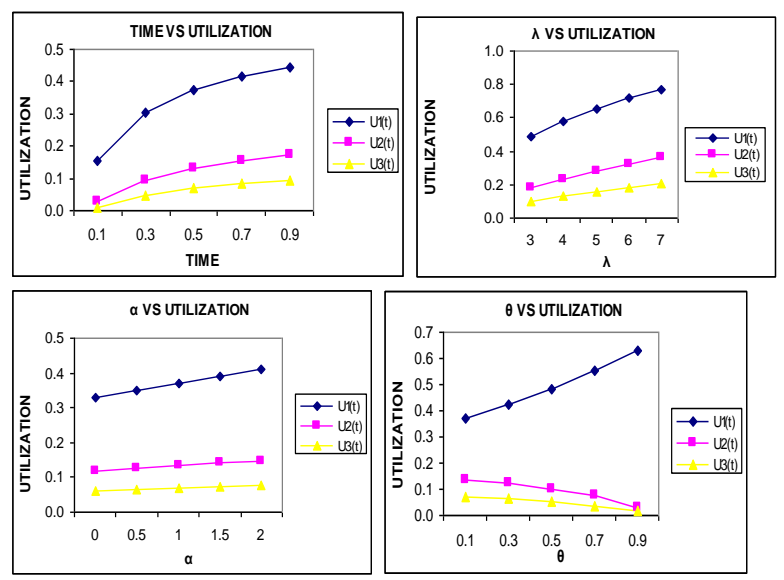

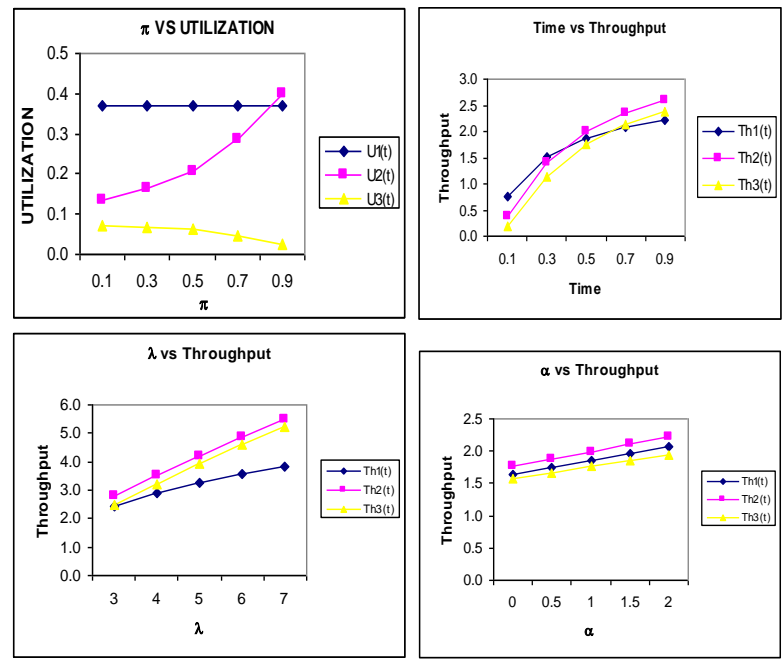
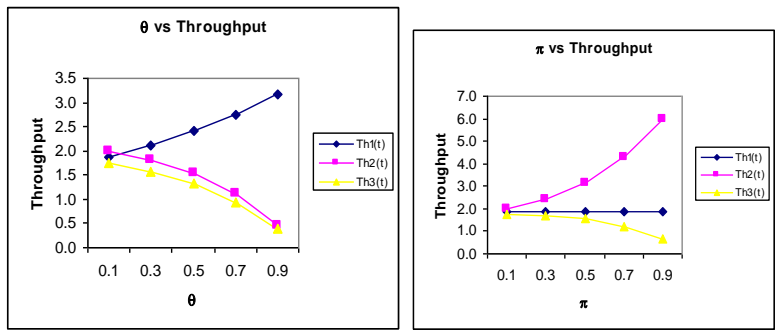

Figure 2: The relationship between Utilization and Throughput and other parameters

Using equations $3.8,3.13,3.18,3.23$ and $3.12,3.17,3.22$, the mean no. of packets in the three buffers and in the network, mean delay in transmission of the three transmitters are calculated for different values of $\mathrm{t}, \lambda, \alpha, \theta, \pi, \mu_{1}, \mu_{2}, \mu_{3}$ and the results are shown in the Table 4.2. The graphs showing the relationship between parameters and performance measure are shown in the Figure 4.2.

Table 4.2 Values of mean number of packets and mean delay of the network model with DBA and Non-Homogeneous arrivals

\begin{tabular}{|c|c|c|c|c|c|c|c|c|c|c|c|c|c|}
\hline $\mathrm{t}$ & $\lambda$ & $\alpha$ & $\theta$ & $\pi$ & $\mu 1$ & $\mu 2$ & $\mu 3$ & L1(t) & L2(t) & L3(t) & $\mathrm{W} 1(\mathrm{t})$ & $\mathrm{W} 2(\mathrm{t})$ & $\mathrm{W} 3(\mathrm{t})$ \\
\hline 0.1 & 2 & 1 & 0.1 & 0.1 & 5 & 15 & 25 & 0.1654 & 0.0261 & 0.0077 & 0.2170 & 0.0675 & 0.0402 \\
\hline 0.3 & 2 & 1 & 0.1 & 0.1 & 5 & 15 & 25 & 0.3593 & 0.0985 & 0.0463 & 0.2381 & 0.0700 & 0.0409 \\
\hline 0.5 & 2 & 1 & 0.1 & 0.1 & 5 & 15 & 25 & 0.4645 & 0.1425 & 0.0729 & 0.2500 & 0.0715 & 0.0415 \\
\hline 0.7 & 2 & 1 & 0.1 & 0.1 & 5 & 15 & 25 & 0.5337 & 0.1696 & 0.0890 & 0.2581 & 0.0725 & 0.0418 \\
\hline 0.9 & 2 & 1 & 0.1 & 0.1 & 5 & 15 & 25 & 0.5882 & 0.1894 & 0.1003 & 0.2646 & 0.0732 & 0.0420 \\
\hline 0.5 & 3 & 1 & 0.1 & 0.1 & 5 & 15 & 25 & 0.6633 & 0.2049 & 0.1053 & 0.2736 & 0.0737 & 0.0421 \\
\hline 0.5 & 4 & 1 & 0.1 & 0.1 & 5 & 15 & 25 & 0.8621 & 0.2673 & 0.1376 & 0.2985 & 0.0760 & 0.0428 \\
\hline 0.5 & 5 & 1 & 0.1 & 0.1 & 5 & 15 & 25 & 1.0609 & 0.3297 & 0.1700 & 0.3245 & 0.0783 & 0.0435 \\
\hline 0.5 & 6 & 1 & 0.1 & 0.1 & 5 & 15 & 25 & 1.2597 & 0.3921 & 0.2023 & 0.3517 & 0.0806 & 0.0442 \\
\hline 0.5 & 7 & 1 & 0.1 & 0.1 & 5 & 15 & 25 & 1.4585 & 0.4545 & 0.2346 & 0.3801 & 0.0830 & 0.0449 \\
\hline 0.5 & 2 & 0 & 0.1 & 0.1 & 5 & 15 & 25 & 0.3976 & 0.1248 & 0.0647 & 0.2424 & 0.0709 & 0.0413 \\
\hline 0.5 & 2 & 0.5 & 0.1 & 0.1 & 5 & 15 & 25 & 0.4311 & 0.1337 & 0.0688 & 0.2462 & 0.0712 & 0.0414 \\
\hline 0.5 & 2 & 1 & 0.1 & 0.1 & 5 & 15 & 25 & 0.4645 & 0.1425 & 0.0729 & 0.2500 & 0.0715 & 0.0415 \\
\hline 0.5 & 2 & 1.5 & 0.1 & 0.1 & 5 & 15 & 25 & 0.4980 & 0.1513 & 0.0771 & 0.2539 & 0.0718 & 0.0416 \\
\hline 0.5 & 2 & 2 & 0.1 & 0.1 & 5 & 15 & 25 & 0.5315 & 0.1602 & 0.0812 & 0.2578 & 0.0721 & 0.0416 \\
\hline 0.5 & 2 & 1 & 0.1 & 0.1 & 5 & 15 & 25 & 0.4645 & 0.1425 & 0.0729 & 0.2500 & 0.0715 & 0.0415 \\
\hline 0.5 & 2 & 1 & 0.3 & 0.1 & 5 & 15 & 25 & 0.5475 & 0.1288 & 0.0654 & 0.2597 & 0.0711 & 0.0413 \\
\hline 0.5 & 2 & 1 & 0.5 & 0.1 & 5 & 15 & 25 & 0.6566 & 0.1084 & 0.0545 & 0.2728 & 0.0703 & 0.0411 \\
\hline 0.5 & 2 & 1 & 0.7 & 0.1 & 5 & 15 & 25 & 0.8023 & 0.0779 & 0.0386 & 0.2908 & 0.0693 & 0.0408 \\
\hline 0.5 & 2 & 1 & 0.9 & 0.1 & 5 & 15 & 25 & 1.0000 & 0.0316 & 0.0154 & 0.3164 & 0.0677 & 0.0403 \\
\hline 0.5 & 2 & 1 & 0.1 & 0.1 & 5 & 15 & 25 & 0.4645 & 0.1425 & 0.0729 & 0.2500 & 0.0715 & 0.0415 \\
\hline 0.5 & 2 & 1 & 0.1 & 0.3 & 5 & 15 & 25 & 0.4645 & 0.1773 & 0.0702 & 0.2500 & 0.0728 & 0.0414 \\
\hline 0.5 & 2 & 1 & 0.1 & 0.5 & 5 & 15 & 25 & 0.4645 & 0.2322 & 0.0651 & 0.2500 & 0.0747 & 0.0413 \\
\hline 0.5 & 2 & 1 & 0.1 & 0.7 & 5 & 15 & 25 & 0.4645 & 0.3370 & 0.0492 & 0.2500 & 0.0785 & 0.0410 \\
\hline 0.5 & 2 & 1 & 0.1 & 0.9 & 5 & 15 & 25 & 0.4645 & 0.5067 & 0.0272 & 0.2500 & 0.0850 & 0.0405 \\
\hline 0.5 & 2 & 1 & 0.1 & 0.1 & 5 & 15 & 25 & 0.4645 & 0.1425 & 0.0729 & 0.2500 & 0.0715 & 0.0415 \\
\hline 0.5 & 2 & 1 & 0.1 & 0.1 & 6 & 15 & 25 & 0.4061 & 0.1510 & 0.0778 & 0.2028 & 0.0718 & 0.0416 \\
\hline 0.5 & 2 & 1 & 0.1 & 0.1 & 7 & 15 & 25 & 0.3591 & 0.1571 & 0.0813 & 0.1700 & 0.0720 & 0.0416 \\
\hline 0.5 & 2 & 1 & 0.1 & 0.1 & 8 & 15 & 25 & 0.3209 & 0.1615 & 0.0840 & 0.1461 & 0.0722 & 0.0417 \\
\hline 0.5 & 2 & 1 & 0.1 & 0.1 & 9 & 15 & 25 & 0.2894 & 0.1647 & 0.0860 & 0.1280 & 0.0723 & 0.0417 \\
\hline 0.5 & 2 & 1 & 0.1 & 0.1 & 5 & 15 & 25 & 0.4645 & 0.1425 & 0.0729 & 0.2500 & 0.0715 & 0.0415 \\
\hline 0.5 & 2 & 1 & 0.1 & 0.1 & 5 & 16 & 25 & 0.4645 & 0.1345 & 0.0735 & 0.2500 & 0.0668 & 0.0415 \\
\hline 0.5 & 2 & 1 & 0.1 & 0.1 & 5 & 17 & 25 & 0.4645 & 0.1273 & 0.0740 & 0.2500 & 0.0626 & 0.0415 \\
\hline 0.5 & 2 & 1 & 0.1 & 0.1 & 5 & 18 & 25 & 0.4645 & 0.1208 & 0.0744 & 0.2500 & 0.0590 & 0.0415 \\
\hline 0.5 & 2 & 1 & 0.1 & 0.1 & 5 & 19 & 25 & 0.4645 & 0.1149 & 0.0748 & 0.2500 & 0.0557 & 0.0415 \\
\hline 0.5 & 2 & 1 & 0.1 & 0.1 & 5 & 15 & 25 & 0.4645 & 0.1425 & 0.0729 & 0.2500 & 0.0715 & 0.0415 \\
\hline 0.5 & 2 & 1 & 0.1 & 0.1 & 5 & 15 & 26 & 0.4645 & 0.1425 & 0.0703 & 0.2500 & 0.0715 & 0.0398 \\
\hline 0.5 & 2 & 1 & 0.1 & 0.1 & 5 & 15 & 27 & 0.4645 & 0.1425 & 0.0678 & 0.2500 & 0.0715 & 0.0383 \\
\hline 0.5 & 2 & 1 & 0.1 & 0.1 & 5 & 15 & 28 & 0.4645 & 0.1425 & 0.0655 & 0.2500 & 0.0715 & 0.0369 \\
\hline 0.5 & 2 & 1 & 0.1 & 0.1 & 5 & 15 & 29 & 0.4645 & 0.1425 & 0.0634 & 0.2500 & 0.0715 & 0.0356 \\
\hline
\end{tabular}


It is observed from the Table 4.2 that as the time $(\mathrm{t})$ varies from 0.1 to 0.9 seconds, the mean number of packets in the three buffers and in the network increases when other parameters are kept constant. When the $\lambda$ changes from $3 \times 10^{4}$ packets/second to $7 \times 10^{4}$ packets/second the mean number of packets in the first, second, third buffers and in the network are increasing. As the parameter $\alpha \square$ increases from 0 to 2 the mean number of packets in three transmitters increases. As the probability parameter $\theta$ varies from 0.1 to 0.9 , the mean number packets in the first buffer increases and decreases in the second and third buffer due to feedback for the first and second buffer. When the second probability parameter $\pi$ varies from 0.1 to 0.9 , the mean number packets in the first buffer remains constant and increases in the second buffer due to packets arrived directly from the first transmitter, decreases in the third buffer due to feedback from the second transmitter. When the transmission rate of the first transmitter $\left(\mu_{1}\right)$ varies from $5 \times 10^{4}$ packets/second to $9 \times 10^{4}$ packets/second, the mean number of packets in the first buffer decreases, in the second and third buffer increases. When the transmission rate of the second transmitter $\left(\mu_{2}\right)$ varies from $15 \times 10^{4}$ packets/second to $19 \times 10^{4}$ packets/second, the mean number of packets in the first buffer remains constant and decreases in the second buffer and increases in the third buffer. When the transmission rate of the third transmitter $\left(\mu_{3}\right)$ varies from $25 \times 10^{4}$ packets/second to $29 \times 10^{4}$ the mean number of packets in the first and second buffer remains constant and decreases in the third buffer.

From the table 4.2, it is also observed that with time (t) and $\lambda$, the mean delay in the three buffers are increasing for fixed values of other parameters. When the parameter $\alpha \square$ increases from 0 to 2, the mean delay in the three buffers also increases. As the parameter $\theta$ varies the mean delay in the first buffer increases and decreases in the second and third buffer due to feedback for the first and second buffer. As the parameter $\pi$ varies the mean delay in the first buffer remains constant and increases in the second buffer and decreases in third buffer. As the transmission rate of the first transmitter $\left(\mu_{1}\right)$ varies, the mean delay of the first buffer decreases, in the second, Third buffer slightly increases. When the transmission rate of the second transmitter $\left(\mu_{2}\right)$ varies, the mean delay of the first and third buffer remains constant and decreases for the second buffer. When the transmission rate of the third transmitter $\left(\mu_{3}\right)$ varies, the mean delay of the first and second buffer remains constant and decreases for the third buffer.

From the above analysis, it is observed that the dynamic bandwidth allocation strategy has a significant influence on all performance measures of the network. We also observed that the performance measures are highly sensitive towards smaller values of time. Hence, it is optimal to consider dynamic bandwidth allocation and evaluate the performance under transient conditions. It is also to be observed that the congestion in buffers and delays in transmission can be reduced to a minimum level by adopting dynamic bandwidth

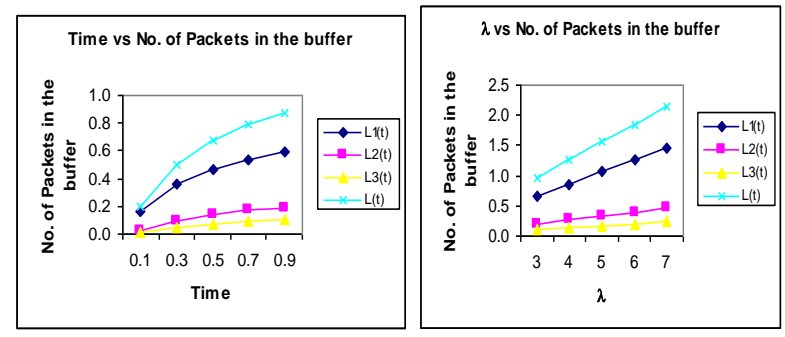

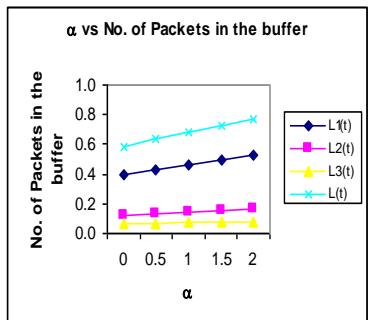
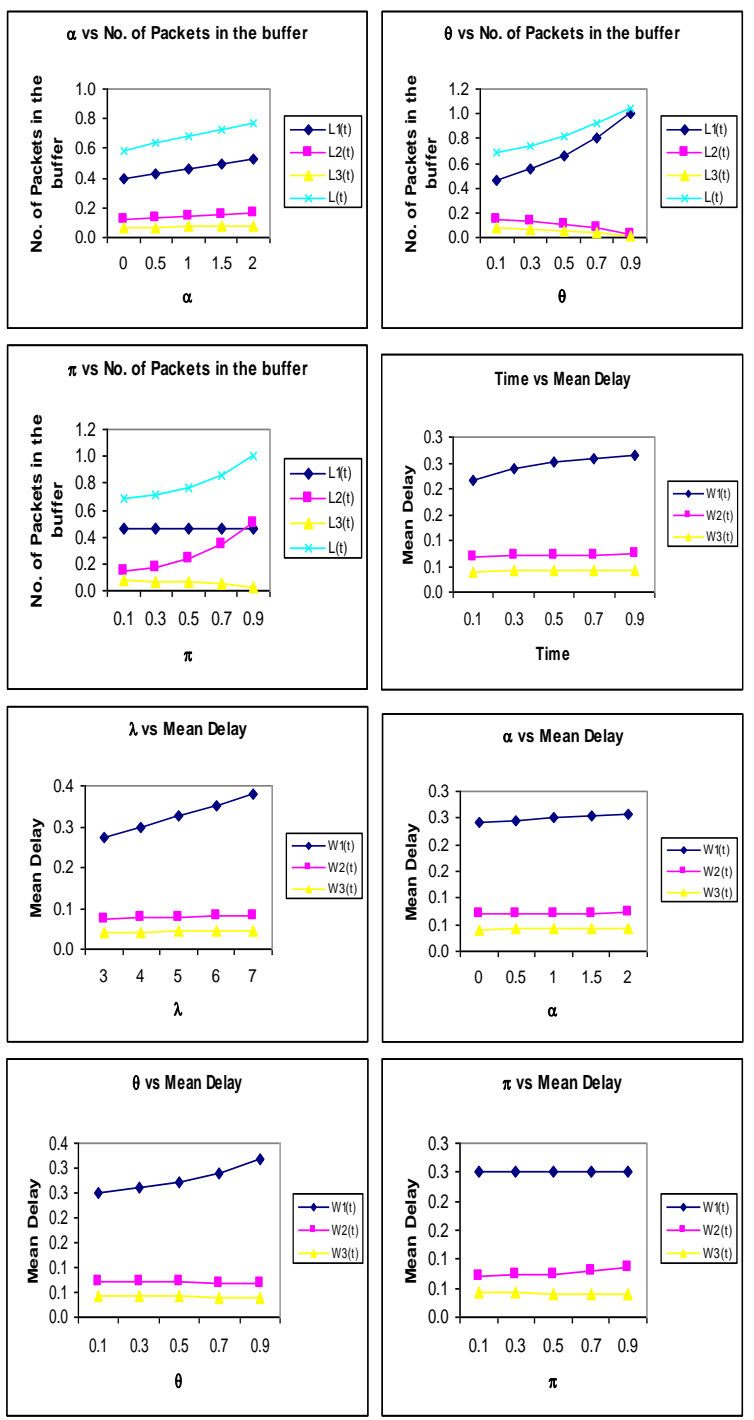

Figure 3: The relationship between mean no. of packets, mean delay and various parameters

\section{SENSITIVITY ANALYSIS}

Sensitivity analysis of the proposed network model with respect to the changes in the parameters $t, \lambda, \alpha, \theta$ and $\pi$ on the mean number of packets, utilization of the transmitters, mean delay and throughput of the three transmitters is presented in this section. The values considered for the sensitivity analysis are, $\mathrm{t}=0.5 \mathrm{sec}, \lambda=2 \times 10^{4}$ packets $/ \mathrm{sec}, \mu_{1}=5 \times 10^{4}$ packets/second, $\quad \mu_{2}=15 \times 10^{4}$ packets $/$ second, $\mu_{3}=25 \times 10^{4}$ packets/second, $\alpha=1, \theta=0.1$ and $\pi=0.1$. The mean number of packets, utilization of the transmitters, mean delay and throughput of the transmitters are computed with variation of $15 \%,-10 \%,-5 \%, 0 \%,+5 \%,+10 \%,+15 \%$ on the model and are presented in the table 5.1. The performance measures are highly affected by the changes in the values of time ( $\mathrm{t}$ ), arrival and probability constants $(\theta, \pi)$. When the time $(\mathrm{t})$ increases from $-15 \%$ to $+15 \%$ the average number of packets in the three buffers increase along with the utilization, throughput of the transmitters and the average delay in buffers. As the arrival parameter $(\lambda)$ increases from $-15 \%$ to $+15 \%$ the average number of packets in the three buffers increase along with the utilization, throughput of the transmitters and the average delay in buffers. When the parameter $\alpha$ varies from $15 \%$ to $+15 \%$, the average number of packets in the three 
buffers increase along with utilization, throughput of the transmitters and the average delay in buffers. As the probability parameter $\theta$ increases from $-15 \%$ to $+15 \%$ the average number of packets in the first buffer increase along with the utilization, throughput of the transmitters and the average delay in buffers. But average number of packets in the second and third buffer decrease along with the utilization, throughput of the transmitter and the average delay in buffer due to feedback for the first and second transmitter. Similarly, when the probability parameter $\pi$ increases from $-15 \%$ to $+15 \%$ the average number of packets, utilization, throughput and the average delay in first buffer remains constant. But average number of packets in the second buffer increase along with the utilization, and the average number of packets in the third buffer decreases along with the utilization, throughput of the transmitter and the average delay in the buffer due to the feedback to the second buffer.

From the above analysis it is observed that the dynamic bandwidth allocation strategy has an important influence on all performance measures of the network. It is also observed that these performance measures are also sensitive towards the probability parameters $(\theta, \pi)$, which causes feedback of packets to the first and second transmitters.

Table 5.1 Sensitive Analysis

\begin{tabular}{|c|c|c|c|c|c|c|c|c|}
\hline \multirow{2}{*}{ Parameter } & \multirow{2}{*}{$\begin{array}{l}\text { Performa } \\
\text { nce } \\
\text { Measure }\end{array}$} & \multicolumn{7}{|c|}{$\%$ change in Parameter } \\
\hline & & -15 & -10 & -5 & 0 & 5 & 10 & 15 \\
\hline \multirow{12}{*}{$\mathrm{t}=0.5$} & $\mathrm{~L}_{1}(\mathrm{t})$ & 0.43115 & 0.44292 & 0.45402 & 0.46453 & 0.47452 & 0.48403 & 0.49313 \\
\hline & $\mathrm{L}_{2}(\mathrm{t})$ & 0.12873 & 0.13362 & 0.13820 & 0.14250 & 0.14654 & 0.15036 & 0.15397 \\
\hline & L3(t) & 0.06460 & 0.06757 & 0.07034 & 0.07294 & 0.07537 & 0.07765 & 0.07980 \\
\hline & $\mathrm{U}_{1}(\mathrm{t})$ & 0.35024 & 0.35784 & 0.36493 & 0.37157 & 0.37782 & 0.38371 & 0.38929 \\
\hline & $\mathrm{U}_{2}(\mathrm{t})$ & 0.12079 & 0.12508 & 0.12908 & 0.13281 & 0.13631 & 0.13960 & 0.14270 \\
\hline & $\mathrm{U} 3(\mathrm{t})$ & 0.06256 & 0.06534 & 0.06793 & 0.07034 & 0.07260 & 0.07471 & 0.07670 \\
\hline & $\mathrm{Th}_{1}(\mathrm{t})$ & 1.75120 & 1.78919 & 1.82465 & 1.85786 & 1.88908 & 1.91854 & 1.94643 \\
\hline & $\mathrm{Th}_{2}(\mathrm{t})$ & 1.81189 & 1.87623 & 1.93618 & 1.99220 & 2.04469 & 2.09401 & 2.14050 \\
\hline & Th3(t) & 1.56401 & 1.63345 & 1.69813 & 1.75848 & 1.81491 & 1.86779 & 1.91749 \\
\hline & $\mathrm{W}_{1}(\mathrm{t})$ & 0.24620 & 0.24755 & 0.24883 & 0.25004 & 0.25119 & 0.25229 & 0.25335 \\
\hline & $\mathrm{W}_{2}(\mathrm{t})$ & 0.07105 & 0.07122 & 0.07138 & 0.07153 & 0.07167 & 0.07180 & 0.07193 \\
\hline & W3(t) & 0.04131 & 0.04137 & 0.04142 & 0.04148 & 0.04153 & 0.04157 & 0.04162 \\
\hline \multirow{12}{*}{$\lambda=2$} & $\mathrm{~L}_{1}(\mathrm{t})$ & 0.40489 & 0.42477 & 0.44465 & 0.46453 & 0.48441 & 0.50429 & 0.52417 \\
\hline & $\mathrm{L}_{2}(\mathrm{t})$ & 0.12378 & 0.13002 & 0.13626 & 0.14250 & 0.14874 & 0.15498 & 0.16122 \\
\hline & L3(t) & 0.06323 & 0.06647 & 0.06970 & 0.07294 & 0.07617 & 0.07940 & 0.08264 \\
\hline & $\mathrm{U}_{1}(\mathrm{t})$ & 0.33295 & 0.34608 & 0.35895 & 0.37157 & 0.38394 & 0.39607 & 0.40796 \\
\hline & $\mathrm{U}_{2}(\mathrm{t})$ & 0.11643 & 0.12192 & 0.12738 & 0.13281 & 0.13821 & 0.14357 & 0.14890 \\
\hline & $\mathrm{U} 3(\mathrm{t})$ & 0.06128 & 0.06431 & 0.06733 & 0.07034 & 0.07334 & 0.07633 & 0.07931 \\
\hline & $\mathrm{Th}_{1}(\mathrm{t})$ & 1.66476 & 1.73041 & 1.79477 & 1.85786 & 1.91971 & 1.98034 & 2.03978 \\
\hline & $\mathrm{Th}_{2}(\mathrm{t})$ & 1.74638 & 1.82883 & 1.91077 & 1.99220 & 2.07313 & 2.15355 & 2.23347 \\
\hline & Th3(t) & 1.53191 & 1.60768 & 1.68320 & 1.75848 & 1.83352 & 1.90832 & 1.98287 \\
\hline & $\mathrm{W}_{1}(\mathrm{t})$ & 0.24321 & 0.24548 & 0.24775 & 0.25004 & 0.25234 & 0.25465 & 0.25698 \\
\hline & $\mathrm{W}_{2}(\mathrm{t})$ & 0.07088 & 0.07109 & 0.07131 & 0.07153 & 0.07175 & 0.07197 & 0.07219 \\
\hline & $\mathrm{W} 3(\mathrm{t})$ & 0.04128 & 0.04134 & 0.04141 & 0.04148 & 0.04154 & 0.04161 & 0.04168 \\
\hline \multirow{12}{*}{$\alpha=1$} & $\mathrm{~L}_{1}(\mathrm{t})$ & 0.45449 & 0.45784 & 0.46119 & 0.46453 & 0.46788 & 0.47123 & 0.47457 \\
\hline & $\mathrm{L}_{2}(\mathrm{t})$ & 0.13985 & 0.14073 & 0.14162 & 0.14250 & 0.14339 & 0.14427 & 0.14515 \\
\hline & L3(t) & $\begin{array}{l}0.07170 \\
\end{array}$ & 0.07211 & 0.07252 & 0.07294 & 0.07335 & 0.07376 & 0.07417 \\
\hline & $\mathrm{U}_{1}(\mathrm{t})$ & 0.36523 & 0.36735 & 0.36947 & 0.37157 & 0.37367 & 0.37576 & 0.37785 \\
\hline & $\mathrm{U}_{2}(\mathrm{t})$ & 0.13051 & 0.13128 & 0.13205 & 0.13281 & 0.13358 & 0.13435 & 0.13511 \\
\hline & $\mathrm{U} 3(\mathrm{t})$ & 0.06919 & 0.06957 & 0.06996 & 0.07034 & 0.07072 & 0.07111 & 0.07149 \\
\hline & $\mathrm{Th}_{1}(\mathrm{t})$ & 1.82615 & 1.83676 & 1.84733 & 1.85786 & 1.86836 & 1.87882 & 1.88925 \\
\hline & $\mathrm{Th}_{2}(\mathrm{t})$ & 1.95764 & 1.96917 & 1.98069 & 1.99220 & 2.00370 & 2.01519 & 2.02667 \\
\hline & Th3(t) & 1.72968 & 1.73928 & 1.74889 & 1.75848 & 1.76808 & 1.77767 & 1.78726 \\
\hline & $\mathrm{W}_{1}(\mathrm{t})$ & 0.24888 & 0.24927 & 0.24965 & 0.25004 & 0.25042 & 0.25081 & 0.25120 \\
\hline & $\mathrm{W}_{2}(\mathrm{t})$ & 0.07144 & 0.07147 & 0.07150 & 0.07153 & 0.07156 & 0.07159 & 0.07162 \\
\hline & $\mathrm{W} 3(\mathrm{t})$ & 0.04145 & 0.04146 & 0.04147 & 0.04148 & 0.04148 & 0.04149 & 0.04150 \\
\hline \multirow{12}{*}{$\theta=0.1$} & $\mathrm{~L}_{1}(\mathrm{t})$ & 0.45914 & 0.46093 & 0.46272 & 0.46453 & 0.46635 & 0.46819 & 0.47003 \\
\hline & $\mathrm{L}_{2}(\mathrm{t})$ & 0.14333 & 0.14306 & 0.14278 & 0.14250 & 0.14222 & 0.14194 & 0.14165 \\
\hline & L3(t) & 0.07340 & 0.07325 & 0.07309 & 0.07294 & 0.07278 & 0.07262 & 0.07246 \\
\hline & $\mathrm{U}_{1}(\mathrm{t})$ & 0.36817 & 0.36930 & 0.37043 & 0.37157 & 0.37272 & 0.37386 & 0.37502 \\
\hline & $\mathrm{U}_{2}(\mathrm{t})$ & 0.13353 & 0.13329 & 0.13306 & 0.13281 & 0.13257 & 0.13232 & 0.13207 \\
\hline & $\mathrm{U} 3(\mathrm{t})$ & 0.07077 & 0.07063 & 0.07048 & 0.07034 & 0.07019 & 0.07004 & 0.06989 \\
\hline & $\mathrm{Th}_{1}(\mathrm{t})$ & 1.84087 & 1.84651 & 1.85217 & 1.85786 & 1.86358 & 1.86932 & 1.87509 \\
\hline & $\mathrm{Th}_{2}(\mathrm{t})$ & 2.00297 & 1.99942 & 1.99583 & 1.99220 & 1.98854 & 1.98484 & 1.98111 \\
\hline & Th3(t) & 1.76930 & 1.76573 & 1.76212 & 1.75848 & 1.75481 & 1.75111 & 1.74737 \\
\hline & $\mathrm{W}_{1}(\mathrm{t})$ & 0.24942 & 0.24962 & 0.24983 & 0.25004 & 0.25025 & 0.25046 & 0.25067 \\
\hline & $\mathrm{W}_{2}(\mathrm{t})$ & 0.07156 & 0.07155 & 0.07154 & 0.07153 & 0.07152 & 0.07151 & 0.07150 \\
\hline & W & 0.04149 & 148 & 148 & 148 & 4147 & 84147 & 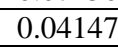 \\
\hline
\end{tabular}




\begin{tabular}{|c|c|c|c|c|c|c|c|c|}
\hline \multirow{12}{*}{$\pi=0.1$} & $\mathrm{~L}_{1}(\mathrm{t})$ & 0.46453 & 0.46453 & 0.46453 & 0.46453 & 0.46453 & 0.46453 & 0.46453 \\
\hline & $\mathrm{L}_{2}(\mathrm{t})$ & 0.14041 & 0.14110 & 0.14180 & 0.14250 & 0.14321 & 0.14393 & 0.14465 \\
\hline & L3(t) & 0.07308 & 0.07304 & 0.07299 & 0.07294 & 0.07288 & 0.07283 & 0.07278 \\
\hline & $\mathrm{U}_{1}(\mathrm{t})$ & 0.37157 & 0.37157 & 0.37157 & 0.37157 & 0.37157 & 0.37157 & 0.37157 \\
\hline & $\mathrm{U}_{2}(\mathrm{t})$ & 0.13100 & 0.13160 & 0.13220 & 0.13281 & 0.13343 & 0.13405 & 0.13468 \\
\hline & $\mathrm{U} 3(\mathrm{t})$ & 0.07048 & 0.07043 & 0.07039 & 0.07034 & 0.07029 & 0.07024 & 0.07020 \\
\hline & $\mathrm{Th}_{1}(\mathrm{t})$ & 1.85786 & 1.85786 & 1.85786 & 1.85786 & 1.85786 & 1.85786 & 1.85786 \\
\hline & $\mathrm{Th}_{2}(\mathrm{t})$ & 1.96497 & 1.97397 & 1.98304 & 1.99220 & 2.00144 & 2.01076 & 2.02017 \\
\hline & Th3(t) & 1.76195 & 1.76081 & 1.75965 & 1.75848 & 1.75730 & 1.75610 & 1.75488 \\
\hline & $\mathrm{W}_{1}(\mathrm{t})$ & 0.25004 & 0.25004 & 0.25004 & 0.25004 & 0.25004 & 0.25004 & 0.25004 \\
\hline & $\mathrm{W}_{2}(\mathrm{t})$ & 0.07146 & 0.07148 & 0.07150 & 0.07153 & 0.07155 & 0.07158 & 0.07160 \\
\hline & W3(t) & 0.04148 & 0.04148 & 0.04148 & 0.04148 & 0.04148 & 0.04147 & 0.04147 \\
\hline
\end{tabular}

\section{COMPARATIVE STUDY}

A comparative study between the performance measures of the network model with non homogeneous Poisson arrivals and Poisson arrivals is performed. The table 6.1 presents the performance measures of both models with fixed values of the parameters $\mathrm{t}, \lambda, \alpha, \theta, \pi, \mu_{1}, \mu_{2}$ and different value of time $\mathrm{t}=$ $0.1,0.3$ seconds. From the table 6.1 it can be observed that as time increases from 0.1 seconds to 0.3 seconds, the percentage of variation of the performance measures between the two network models also increases. The network model with nonhomogeneous Poisson arrivals and dynamic bandwidth allocation has higher utilization than the network model with homogeneous compound Poisson arrivals. It can also be observed that non-homogeneous Poisson arrivals have a significant influence on all the performance measures of the network model.

Table 6.1Comparative study of the network model withnon homogeneous and homogeneous Poisson arrivals

\begin{tabular}{|c|c|c|c|c|c|}
\hline $\mathrm{T}$ & Parameters & $\alpha=1$ & $\alpha=0$ & Difference & $\begin{array}{c}\% \text { of } \\
\text { variation }\end{array}$ \\
\hline \multirow{12}{*}{0.1} & $\mathrm{~L} 1(\mathrm{t})$ & 0.165381 & 0.161054 & 0.004327 & 2.6869 \\
\hline & $\mathrm{L} 2(\mathrm{t})$ & 0.026148 & 0.025656 & 0.000492 & 1.9185 \\
\hline & $\mathrm{L} 3(\mathrm{t})$ & 0.0077 & 0.007539 & 0.000115 & 1.5256 \\
\hline & $\mathrm{U} 1(\mathrm{t})$ & 0.152430 & 0.148754 & 0.003676 & 2.4710 \\
\hline & $\mathrm{U} 2(\mathrm{t})$ & 0.025809 & 0.025330 & 0.000480 & 1.8936 \\
\hline & $\mathrm{U} 3(\mathrm{t})$ & 0.007625 & 0.007511 & 0.000114 & 1.5198 \\
\hline & $\mathrm{TH} 1(\mathrm{t})$ & 0.762148 & 0.743770 & 0.018378 & 2.4710 \\
\hline & $\mathrm{TH} 2(\mathrm{t})$ & 0.387139 & 0.379945 & 0.007194 & 1.8936 \\
\hline & $\mathrm{TH} 3(\mathrm{t})$ & 0.190620 & 0.187767 & 0.002854 & 1.5198 \\
\hline & $\mathrm{W} 1(\mathrm{t})$ & 0.216994 & 0.216538 & 0.000456 & 0.2107 \\
\hline & $\mathrm{W} 2(\mathrm{t})$ & 0.067542 & 0.067526 & 0.000017 & 0.0245 \\
\hline & $\mathrm{W} 3(\mathrm{t})$ & 0.040153 & 0.040151 & 0.000002 & 0.0057 \\
\hline \multirow{11}{*}{0.3} & $\mathrm{~L} 1(\mathrm{t})$ & 0.359312 & 0.329227 & 0.030086 & 9.1384 \\
\hline & $\mathrm{L} 2(\mathrm{t})$ & 0.098457 & 0.091830 & 0.006628 & $\begin{array}{r}7.2172 \\
\end{array}$ \\
\hline & L3 (t) & 0.04627 & 0.043566 & 0.002708 & 6.2148 \\
\hline & $\mathrm{U} 1(\mathrm{t})$ & 0.301844 & 0.280520 & 0.021324 & 7.6015 \\
\hline & $\mathrm{U} 2(\mathrm{t})$ & 0.093766 & 0.087740 & 0.006026 & 6.8681 \\
\hline & $\mathrm{U} 3(\mathrm{t})$ & 0.045219 & 0.042631 & 0.002589 & 6.0722 \\
\hline & $\mathrm{TH} 1(\mathrm{t})$ & 1.509219 & 1.402600 & 0.106619 & 7.6015 \\
\hline & $\mathrm{TH} 2(\mathrm{t})$ & 1.406484 & 1.316093 & 0.090391 & 6.8681 \\
\hline & $\mathrm{TH} 3(\mathrm{t})$ & 1.130481 & 1.065766 & 0.064716 & 6.0722 \\
\hline & $\mathrm{W} 1(\mathrm{t})$ & 0.238078 & 0.234726 & 0.003352 & 1.4283 \\
\hline & $\mathrm{W} 2(\mathrm{t})$ & 0.070002 & 0.069775 & 0.000228 & 0.3267 \\
\hline
\end{tabular}

\section{CONCLUSION}

This paper introduces a tandem communication network model with three transmitters with dynamic bandwidth allocation and feedback for first and second transmitters. Arrival of packets at the buffers follows non homogeneous Poisson arrivals and dynamic bandwidth allocation at the transmitters. The non homogeneous Poisson arrivals can model the time dependent nature of burst arrivals. The dynamic bandwidth allocation is adapted by immediate adjustment of packet service time by utilizing idle bandwidth in the transmitter. A numerical study reveals the that this communication model is capable of predicting the performance measures of the network like average content of the buffers, mean delays, throughput of the transmitters, idleness of the transmitters etc, explicitly. It is observed that the feedback probability parameters $(\theta, \pi)$ have significant influence on the overall performance of the network. The sensitivity analysis of network reveals that the dynamic Bandwidth allocation strategy can reduce the congestion in buffers and mean delay in transmission.

\section{REFERENCES}

[1] S. Balsamo, V.D.N. Persone, P. Inverardi, A review on queueing network models with finite capacity queues for software architectures performance prediction, Perform. Eval. 51 (2003) 269-288.

[2] A. Gomez-Corral, Sojourn times in a two-stage queueing network with blocking, Naval Res. Logist. 51 (2004) 1068-1089.

[3] Avi-Itzhak, B., Yadin, M. A sequence of two serverswith no intermediate queue. Management Sci. 11,(1965) 553564.

[4] Tang, J. and Zhao, Y.Q. "Stationary tail asymptotic of a tandem queue with feedback", Annals of Operation Research, Vol. 160, pp.173-189, 2008

[5] R.D. van der Mei, B.M.M. Gijsen, N. in't Veld, J.L. van der Berg, Response times in a two-node queueing network with feedback, Performance Evaluation 49 (2002) 99-110

[6] Kleinrock L., (1976), Queuing system, vol.II, Computer Applications, wiley publications, Newyork.

[7] Suresh Varma P., K. Srinivas Rao (2007), A Communication Network with load dependent Transmission, International Journal of Mathematical Sciences, vol.6, no.2, pp 199-210. 
[8]Kin K Leung (2002), Load dependent service queues with application to congestion control in broadband Networks, performance evaluation, vol. 50, issue 1-4, pp. 27-40.

[9] Sriram K.(1993), Methodologies for bandwidth allocation, transmission scheduling and congestion control avoidance in broadband ATM Networks, computer Network, ISDN system, j.26, pp. 43-59

[10] M.V.Rama Sundari, K. Srinivasa Rao, P.Srinivasa Rao, P.Suresh Varma, On Tandem Communication Network
Model with DBA having NHP Arrivals for First Node and Poisson process arrivals for Second Node in International Journal of Computer Science (136 - 144) Volume 8 - No.2, September 2011

[11] M.V.Rama Sundari, K. Srinivasa Rao, P.Srinivasa Rao, P.Suresh Varma, Three Node Tandem Communication Network Model with Dynamic Bandwidth Allocation and Non Homogeneous Poisson Arrivals in International Journal of Computer Applications (0975 - 8887) Volume 31- No.1, October 2011 